\title{
FLAT AFFINE AND SYMPLECTIC GEOMETRIES ON LIE GROUPS
}

\author{
VILLABÓN, ANDRÉS.*
}

\begin{abstract}
In this paper we exhibit a family of flat left invariant affine structures on the double Lie group of the oscillator Lie group of dimension 4, associated to each solution of classical YangBaxter equation given by Boucetta and Medina. On the other hand, using Koszul's method, we prove the existence of an immersion of Lie groups between the group of affine transformations of a flat affine and simply connected manifold and the classical group of affine transformations of $\mathbb{R}^{n}$. In the last section, for each flat left invariant affine symplectic connection on the group of affine transformations of the real line, describe for Medina-Saldarriaga-Giraldo, we determine the affine symplectomorphisms. Finally we exhibit the Hess connection, associated to a Lagrangian bi-foliation, which is flat left invariant affine.
\end{abstract}

Keywords: Flat affine Lie groups, Development of flat affine manifold, oscillator Lie group.

* Instituto de Matemáticas, Universidad de Antioquia, Medellín-Colombia e-mails: edgar.villabon@udea.edu.co

\section{INTRODUCTION}

Let $M$ be a (paracompact) connected real smooth manifold of dimension $n$. A linear frame $u$ at point $x \in M$ is an ordered basis $\left(X_{1}, \ldots, X_{n}\right)$ of the tangent space $T_{x}(M)$ it will usually be identified with a linear isomorphism from $\mathbb{R}^{n}$ onto $T_{x}(M)$. The set $L(M)$ of all linear frames at all points of $M$ is a smooth principal fiber bundle over $M$ with group $\operatorname{GL}(n, \mathbb{R})$, the right action of $\mathrm{GL}(n, \mathbb{R})$ on $L(M)$ is given by

$$
u \cdot a=u \circ a \quad \text { with } u \in L(M) \text { and } a \in \mathrm{GL}(n, \mathbb{R}) .
$$

The diffeomorphism of $L(M)$ associated to $a$ will be denoted $R_{a}(u)=u \cdot a$.

The canonical form $\theta$ of $L(M)$ is the $\mathbb{R}^{n}$-valued 1 -form defined by

$$
\theta\left(X_{u}\right):=u^{-1}\left(\pi_{*, u}\left(X_{u}\right)\right) \quad \text { for } X_{u} \in T_{u}(L(M))
$$

where $\pi: L(M) \rightarrow M$ in the natural projection.

In what follows we will privilege the notion of connection due to C. Ehresmann [7]. When necessary we will also use the notions of E. Cartan [2] or J.L. Koszul [12].

A Linear connection $\Gamma$ over $M$ is a smooth distribution $H$ in $L(M)$ transverse and supplementary to the fiber and invariant under the right action of $\mathrm{GL}(n, \mathbb{R})$, defined above. The subspace $H_{u}$, where $u$ is a linear frame at $x \in M$, is called the horizontal space in $u$.

It is clear that every vector $X_{u} \in T_{u}(L(M))$ can be uniquely written as $X_{u}=X_{u, H}+X_{u, V}$, with $X_{u, H} \in H_{u}$ and $X_{u, V}$ tangent to the fiber over $u$.

Each $\vec{x} \in \mathbb{R}^{n}$ determines a standard horizontal vector field $B(\vec{x})_{u}$ defined as the unique

Date: May 30, 2018. 
element of $H_{u}$ such that $\pi_{*, u}\left(B(\vec{x})_{u}\right)=u(\vec{x})$.

Moreover, $\Gamma$ defines a unique 1 -form $\omega$ on $L(M)$ with values in $\mathfrak{g}=\mathrm{gl}(n, \mathbb{R})$, called the connection form. More precisely $\omega_{u}\left(X_{u}\right)$ is the unique $A \in \mathfrak{g}$ such that $A_{u}^{*}=X_{u, V}$ where

$$
A_{u}^{*}=\left.\frac{\mathrm{d}}{\mathrm{dt}}\right|_{t=0} u \cdot \exp t A
$$

is the fundamental vector field corresponding to $A$.

The connection form $\omega$ satisfies the following conditions

$$
\begin{aligned}
\omega\left(A^{*}\right) & =A \quad \text { for all } A \in \mathfrak{g}, \\
\left(R_{\sigma}\right)^{*} \omega & =\operatorname{ad}\left(\sigma^{-1}\right) \omega \quad \text { for all } \sigma \in \mathrm{GL}(n, \mathbb{R}),
\end{aligned}
$$

where ad denotes the adjoint representation of $\mathrm{GL}(n, \mathbb{R})$. Having a connection $\Gamma$ is equivalent to have a 1-form $\omega$ on $L(M)$ with values in $\mathfrak{g}$ verifying (11) y (2). A smooth vector field $X \in \mathfrak{X}(L(M))$ such that $\omega(X)=0$, is called horizontal vector relative to $\Gamma$.

The curvature form $\Omega$ and the torsion form $\Theta$ of $(L(M), \Gamma)$ are defined respectively by

$$
\begin{gathered}
\Omega_{u}\left(X_{u}, Y_{u}\right)=(\mathrm{d} \omega)_{u}\left(X_{u, H}, Y_{u, H}\right) \\
\Theta_{u}\left(X_{u}, Y_{u}\right)=(\mathrm{d} \theta)_{u}\left(X_{u, H}, Y_{u, H}\right)
\end{gathered}
$$

with $X_{u}, Y_{u} \in T_{u}(L(M))$ and $u \in L(M)$.

Note that $\Omega$ takes values in $\mathrm{gl}(n, \mathbb{R})$ and $\Theta$ in $\mathbb{R}^{n}$. The following equations are called structure equations (also known as Cartan equations) (see [11]),

$$
\begin{aligned}
(\mathrm{d} \theta)_{u}\left(X_{u}, Y_{u}\right) & =-\frac{1}{2}\left(\omega_{u}\left(X_{u}\right) \theta_{u}\left(Y_{u}\right)-\omega_{u}\left(Y_{u}\right) \theta_{u}\left(X_{u}\right)\right)+\Theta_{u}\left(X_{u}, Y_{u}\right), \\
(\mathrm{d} \omega)_{u}\left(X_{u}, Y_{u}\right) & =-\frac{1}{2}\left[\omega_{u}\left(X_{u}\right), \omega\left(Y_{u}\right)\right]+\Omega_{u}\left(X_{u}, Y_{u}\right)
\end{aligned}
$$

Given a global (or local) diffeomorphism $f$ of $M$ we will denote $\tilde{f}$ to the global (or local) diffeomorphism of $L(M)$ defined by $\widetilde{f}(u)=\left(f_{*, x}\left(X_{1}\right), \ldots, f_{*, x}\left(X_{n}\right)\right)$ with $u=\left(X_{1}, \ldots, X_{n}\right)$ a linear frame at $x \in M$. The diffeomorphism $\tilde{f}$ is called the natural lift of $f$ to $L(M)$. When $f$ satisfies the condition $\tilde{f}^{*} \omega=\omega$, it is called a global (or local) affine transformation of $(M, \Gamma)$.

If $M$ is simply connected endowed with a linear connection $\Gamma$, it is well known that in $\Gamma$ is flat, that is, without curvature, the connection over $M$ is isomorphic to the connection $\Gamma^{\prime}$ given by the distribution $H$ in $(M \times \mathrm{GL}(n, \mathbb{R}), \psi)$ where $H_{u}$ is the tangent space to $M \times\{a\}$ in $u=(x, a) \in M \times \mathrm{GL}(n, \mathbb{R})$. The connection $\Gamma^{\prime}$ is called the canonical flat (linear) connection over $M$ (see [11]).

Let $\pi_{2}: M \times \mathrm{GL}(n, \mathbb{R}) \rightarrow \mathrm{GL}(n, \mathbb{R})$ natural projection. Notice that the connection form of the canonical flat linear connection is given by

$$
\omega^{\prime}=\pi_{2}^{*} \theta^{+}
$$

where $\theta^{+}$is the left invariant 1 -form over $\mathrm{GL}(n, \mathbb{R})$, with values in $\mathrm{gl}(n, \mathbb{R})$ defined by

$$
\theta^{+}\left(A^{+}\right):=A^{+}
$$


and $A^{+} \in \mathfrak{g}$ is the left invariant vector field corresponding to $A \in T_{\varepsilon} \mathrm{GL}(n, \mathbb{R})$. The 1 -form $\theta^{+}$ is called the canonical 1 -form over $\mathrm{GL}(n, \mathbb{R})$.

If $M=G$ is a Lie group and the left multiplications $L_{\sigma}$ (respectively right multiplications $R_{\sigma}$ ) are affine transformations it is said that $\Gamma$ is left invariant (respectively right invariant). This means that the lift $\widetilde{L}_{\sigma}$ of $L_{\sigma}$ (respectively $\widetilde{R}_{\sigma}$ ) preserves the distribution $H$ given by $\Gamma$. In this case we denote the connection by $\Gamma^{+}$and we will say that $\left(G, \Gamma^{+}\right)$is an affine Lie group.

It is known that having a linear connection $\Gamma$ over $M$ is equivalent to having a covariant derivative $\nabla$ (see [1] $)$. Let us consider the product

$$
X Y:=\nabla_{X} Y, \text { for } \quad X, Y \in \mathfrak{X}(M) .
$$

If the curvature tensor relative to $\nabla$ is identically zero, this product verifies the condition

$$
[X, Y] Z=X(Y Z)-Y(X Z), \quad \text { for all } \quad X, Y, Z \in \mathfrak{X}(M) .
$$

If both torsion and curvature vanish identically we have

$$
(X Y) Z-X(Y Z)=(Y X) Z-Y(X Z), \text { for all } \quad X, Y, Z \in \mathfrak{X}(M) .
$$

A vector space endowed with a bilinear product satisfying Equation (6) is called a left symmetric algebra. Left symmetric algebras are Lie algebras with the bracket given by $[X, Y]_{1}:=$ $X Y-Y X$. If $\nabla$ is torsion free this Lie bracket agrees with the usual Lie bracket of $\mathfrak{X}(M)$, that is

$$
[X, Y]=X Y-Y X
$$

Recall that product (5) satisfies $(f X) Y=f(X Y)$ and $X(g Y)=X(g) Y+g(X Y)$, for all $f, g \in C^{\infty}(M, \mathbb{R})$, i.e., the product defined above is $\mathbb{R}$-bilinear and $C^{\infty}(M, \mathbb{R})$-linear in the first component.

If $M=G$ is a Lie group and $\nabla=\nabla^{+}$is left invariant, it is well known that $\left(G, \nabla^{+}\right)$is flat affine if and only if Product (5) turn $\mathfrak{g}$ into a left symmetric algebra (see [15]). If this is the case, we denote by $\omega^{+}$the corresponding connection form associated to the connection $\nabla^{+}$.

\section{Flat affine Geometry on 4-Dimensional oscillator Lie Group}

The 3-dimensional Heisenberg Lie group is the real manifold $\mathrm{H}_{3}=\mathbb{R} \times \mathbb{C}$ with product

$$
(s, w)\left(s^{\prime}, w^{\prime}\right)=\left(s+s^{\prime}+\frac{1}{2} \operatorname{lm}\left(\bar{w} w^{\prime}\right), w+w^{\prime}\right)
$$

Definition 2.1. Let $\lambda$ be a positive real number and $\rho: \mathbb{R} \rightarrow \operatorname{Aut}\left(\mathrm{H}_{3}\right)$ the Lie group homomorphism given by

$$
\rho(t)(s, z)=\left(s, e^{i t \lambda} z\right)
$$

The real $\lambda$-oscillator group of dimension 4 , denoted by $O_{\lambda}$, is the semidirect product of Heisenberg Lie group $\mathrm{H}_{3}$ for the group $(\mathbb{R},+)$ by the representation $\rho$.

The following result is known (see [16]).

Lemma 2.1. The Lie group $O_{\lambda}$ is independent of $\lambda$, for $\lambda>0$. 
Proof. The statement follows from the fact that the map $\phi: \mathrm{H}_{3} \rightarrow \mathrm{H}_{3}$ defined by $\phi(s, z)=$ $\left(s, z^{\frac{\lambda^{\prime}}{\lambda}}\right)$, is an automorphism of Lie groups such that for all $t \in \mathbb{R}$ the following diagram commutes

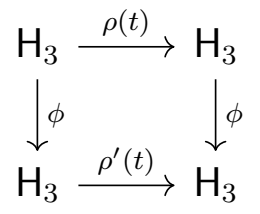

where $\rho$ and $\rho^{\prime}$ are the representations

$$
\begin{aligned}
& \rho: \mathbb{R} \rightarrow \operatorname{Aut}\left(\mathrm{H}_{3}\right) \\
& t \mapsto\left(s, e^{\lambda t i}\right)
\end{aligned}
$$

In the follows we denote by $O$ the oscillator Lie group of dimension 4 with $\lambda=1$. Let $\left(e_{0}, e_{1}, e_{2}, d\right)$ be the natural basis for $\mathbb{R}^{4}$. The corresponding left invariant vector fields are as follows:

$$
\begin{aligned}
e_{0}^{+} & =\frac{\partial}{\partial x_{1}} \\
e_{1}^{+} & =\left(\frac{1}{2}\left(x_{2} \sin \left(x_{4}\right)-x_{3} \cos \left(x_{4}\right)\right)\right) \frac{\partial}{\partial x_{1}}+\cos \left(x_{4}\right) \frac{\partial}{\partial x_{2}}+\sin \left(x_{4}\right) \frac{\partial}{\partial x_{3}} \\
e_{2}^{+} & =\left(\frac{1}{2}\left(x_{2} \cos \left(x_{4}\right)+x_{3} \sin \left(x_{4}\right)\right)\right) \frac{\partial}{\partial x_{1}}-\sin \left(x_{4}\right) \frac{\partial}{\partial x_{2}}+\cos \left(x_{4}\right) \frac{\partial}{\partial x_{3}} \\
d^{+} & =\frac{\partial}{\partial x_{4}} .
\end{aligned}
$$

It is directly verified that the non zero Lie brackets, except antisymmetry, are given by

$$
\left[e_{1}^{+}, e_{2}^{+}\right]=e_{0}^{+}, \quad\left[d^{+}, e_{1}^{+}\right]=e_{2}^{+}, \quad\left[d^{+}, e_{2}^{+}\right]=-e_{1}^{+} .
$$

consequently the Lie algebra of $O$ is the real vector space with basis $\left\{e_{0}^{+}, e_{1}^{+}, e_{2}^{+}, d^{+}\right\}$and bracket defined in (9). This Lie algebra will be denoted by $\mathfrak{o}$.

2.1. Flat affine structures on $O$. To construct these kind of structures, we start by finding flat left invariant affine structures on the group of isometries of the Euclidean plane $E(2)$, called the Euclidean group. Then we use the cohomology of left symmetric algebras developed in [18] to extend these structures to the oscillator group of dimension 4.

Notice that the oscillator group can be viewed as an extension of the Euclidean group E(2). This extension is given by the exact split sequence of Lie groups

$$
\{0\} \longrightarrow(\mathbb{R},+) \stackrel{i}{\longrightarrow} O \stackrel{\pi}{\longrightarrow} E(2) \longrightarrow\{I\}
$$

where $i(s)=(s, 0,0)$, for all $s \in \mathbb{R}$ and

$$
\pi(s, x+i y, t)=\left(\begin{array}{ccc}
\cos (t) & -\sin (t) & x \\
\sin (t) & \cos (t) & y \\
0 & 0 & 1
\end{array}\right), \quad \text { for all }(s, x+i y, t) \in O
$$


and the section is given by the Lie group homomorphism $\mathbf{s}: E(2) \rightarrow O$ defined by $\mathbf{s}(A,(x, y))=$ $(0, x+i y, t)$ where $A=\left(\begin{array}{cc}\cos (t) & -\sin (t) \\ \sin (t) & \cos (t)\end{array}\right)$.

The Lie algebra of the Euclidean Lie group, $\mathfrak{e}(2)=\operatorname{Vect}_{\mathbb{R}}\left\{e_{1}, e_{2}, d\right\}$, has Lie bracket given by $\left[d, e_{1}\right]=e_{2},\left[d, e_{2}\right]=-e_{1}$, and $\left[e_{1}, e_{2}\right]=0$.

Proposition 2.1. The following left symmetric products determine flat left invariant nonisomorphic affine structures on the Euclidean group E(2).

$$
\begin{aligned}
& \begin{array}{c|ccc} 
& e_{1} & e_{2} & d \\
\hline e_{1} & 0 & 0 & \alpha e_{1} \\
e_{2} & 0 & 0 & \alpha e_{2} \\
d & \alpha e_{1}+e_{2} & -e_{1}+\alpha e_{2} & \alpha d
\end{array} \\
& \mathcal{F}_{1}(\alpha)
\end{aligned}
$$

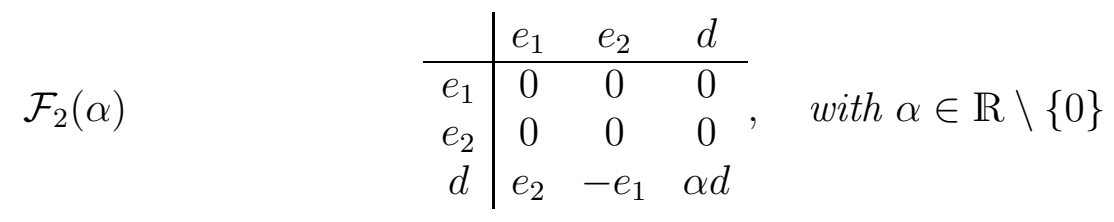

$$
\begin{aligned}
& \begin{array}{cc|ccc} 
& & e_{1} & e_{2} & d \\
\hline \mathcal{F}_{3} & e_{1} & d & 0 & 0 \\
& e_{2} & 0 & d & 0 \\
& d & e_{2} & -e_{1} & 0
\end{array} \\
& \begin{array}{cc|ccc} 
& & e_{1} & e_{2} & d \\
\cline { 2 - 5 } \mathcal{F}_{4} & e_{1} & e_{1}+2 e_{2}-d & 0 & e_{1}+2 e_{2}-d \\
& e_{2} & 0 & e_{1}+2 e_{2}-d & 2 e_{1}+4 e_{2}-2 d \\
& d & e_{1}+3 e_{2}-d & e_{1}+4 e_{2}-2 d & 3 e_{1}+11 e_{2}-5 d
\end{array}
\end{aligned}
$$

Proof. Recall that finding flat left invariant affine structures on $E(2)$ is equivalent to finding bilinear products on $\mathfrak{e}(2)$ satisfying (6) and (7)).

Let us denote by $L_{a}$ the endomorphism of the vector space $\mathfrak{e}(2)$ defined by $L_{a}(b)=a b$.

If a bilinear product is compatible with the Lie bracket of $\mathfrak{e}(2)$, the endomorphisms $L_{e_{1}}, L_{e_{2}}$ and $L_{d}$ can be writen, in the base $\left(e_{1}, e_{2}, d\right)$, as

$$
L_{e_{1}}=\left(a_{i j}\right), \quad L_{e_{2}}=\left(\begin{array}{lll}
a_{12} & b_{12} & b_{13} \\
a_{22} & b_{22} & b_{23} \\
a_{32} & b_{32} & b_{33}
\end{array}\right), \quad L_{d}=\left(\begin{array}{ccc}
a_{13} & b_{13}-1 & c_{13} \\
1+a_{23} & b_{23} & c_{23} \\
a_{33} & b_{33} & c_{33}
\end{array}\right)
$$

where $a_{i j}, b_{i j}$ y $c_{i j}$ are real numbers.

Moreover, if the product on $\mathfrak{e}(2)$ satisfies (6) , the Endomorphisms (14) satisfy the system of equations

$$
\begin{aligned}
{\left[L_{e_{1}}, L_{e_{2}}\right] } & =0 \\
{\left[L_{d}, L_{e_{1}}\right] } & =L_{e_{2}} \\
{\left[L_{d}, L_{e_{2}}\right] } & =-L_{e_{1}}
\end{aligned}
$$

Solving the system and using the above observations we obtain the products described in the tables. 
2.2. Left symmetric algebra cohomology. Let $A$ be a left symmetric algebra and $M$ a $A$-bimodulo. We denote by $H_{S G}^{k}(A, M)$ the cohomology group of order $k$ of $A$ with coefficients in $M$. Let us consider $\mathbb{R}$ as a trivial $A$-bimodulo. A scalar 2-cocycle of $A$ is a bilinear map $f: A \times A \rightarrow \mathbb{R}$ verifying

$$
f(a b-b a, c)=f(a, b c)-f(b, a c) .
$$

A scalar 2-coboundary of $A$ is a 2-cocycle $f$ such that $f(a, b)=\varphi(a b)$, for some $\varphi \in A^{*}$. The cohomology group $H_{S G}^{2}(A, \mathbb{R})=Z^{2} / B^{2}$, where $Z^{2}$ (respectively $B^{2}$ ) is the set of 2-cocycles (respectively 2-coboundaries), classifies the left symmetric algebras extensions of $A$ for which $\mathbb{R}$ is an ideal of null square (see [18]). In particular

$$
0 \rightarrow \mathbb{R} \rightarrow \mathbb{R} \times A \rightarrow A \rightarrow 0
$$

is an exact sequence of left symmetric algebras.

Proposition 2.2. The 2-order cohomology groups of left symmetric algebras of $\mathfrak{e}(2)$ corresponding to the products $\mathcal{F}_{1}(0), \mathcal{F}_{1}(\alpha), \mathcal{F}_{2}(\alpha), \mathcal{F}_{3}$ y $\mathcal{F}_{4}$ are respectively given by

$$
\begin{aligned}
H_{S G, 1,0}^{2}\left(\mathfrak{e}(2), \mathbb{R e}_{0}\right) & \cong \operatorname{Vect}_{\mathbb{R}}\left\{E_{12}-E_{21}, E_{22}+E_{11}, E_{33}\right\} \\
H_{S G, 1, \alpha}^{2}\left(\mathfrak{e}(2), \mathbb{R} e_{0}\right) & \cong\{0\}, \text { si } \alpha \neq 0 \\
H_{S G, 2, \alpha}^{2}\left(\mathfrak{e}(2), \mathbb{R} e_{0}\right) & \cong \operatorname{Vect}_{\mathbb{R}}\left\{E_{11}+E_{22}, E_{12}-E_{21}\right\} \\
H_{S G, 3}^{2}\left(\mathfrak{e}(2), \operatorname{Re}_{0}\right) & \cong \operatorname{Vect}\left\{E_{12}-E_{21}\right\} \\
H_{S G, 4}^{2}\left(\mathfrak{e}(2), \operatorname{Re}_{0}\right) & \cong \operatorname{Vect}\left\{E_{12}-E_{21}+2 E_{13}-E_{23}\right\}
\end{aligned}
$$

where $\left\{E_{i j}\right\}$ denote the elementary matrices on $\mathrm{M}_{3 \times 3}(\mathbb{R})$.

Proof. Let us consider $M=\mathbb{R} e_{0}$ as a trivial $\mathfrak{e}(2)$-bimodule, with $\mathfrak{e}(2)=\operatorname{Vect}_{\mathbb{R}}\left(e_{1}, e_{2}, d\right)$. Each of the following statements can be verified by direct calculations.

In the algebra with product given by $\mathcal{F}_{1}(0)$, the scalar 2-cocycles of $\mathfrak{e}(2)$ with coefficients in $M$, are of the form

$$
g=p\left(E_{11}+E_{22}\right)+q\left(E_{12}-E_{21}\right)+r E_{31}+s E_{32}+t E_{33}
$$

and the 2-coboundaries as

$$
f=r E_{31}+s E_{32} \text {. }
$$

In the algebras corresponding to the products $\mathcal{F}_{1}(\alpha)$, with $\alpha \neq 0$, the 2 -cocycles (respectively the 2-coboundaries), are given by

$$
g=p\left(E_{13}+E_{31}-\frac{1}{\alpha} E_{32}\right)+q\left(E_{23}+\frac{1}{\alpha} E_{31}+E_{32}\right)+r E_{33}
$$

and

$$
f=p\left(\alpha E_{13}+\alpha E_{31}-E_{32}\right)+q\left(\alpha E_{23}+E_{31}+\alpha E_{32}\right)+r E_{33}
$$

In the algebras with product $\mathcal{F}_{2}(\alpha)$ the 2-cocycles (respectively the 2-coboundaries) are written

$$
g=t\left(E_{11}+E_{22}\right)+s\left(E_{12}-E_{21}\right)+r E_{31}+p E_{32}+q E_{33}
$$

and

$$
f=r E_{31}+p E_{32}+q E_{33}
$$


The 2-cocycles (respectively the 2-coboundaries) associated to the algebra with product $\mathcal{F}_{3}$, are given by

and

$$
g=t\left(E_{11}+E_{22}\right)+s\left(E_{12}-E_{21}\right)+r E_{31}+p E_{32}
$$

$$
f=t\left(E_{11}+E_{22}\right)+r E_{31}+p E_{32}
$$

Finally, the scalar 2-cocycles (respectively 2-coboundaries) of the left symmetric algebra defined by $\mathcal{F}_{4}$, are of the form

$$
\begin{gathered}
g=p\left(E_{11}+E_{22}+E_{13}+2 E_{23}\right)+q\left(E_{12}-E_{21}+2 E_{13}-E_{23}\right)+ \\
r\left(E_{31}+E_{33}\right)+s\left(E_{32}+2 E_{33}\right)
\end{gathered}
$$

and

$$
f=p\left(E_{11}+E_{22}+E_{13}+2 E_{23}\right)+r\left(E_{31}+E_{33}\right)+s\left(E_{32}+2 E_{33}\right),
$$

where $p, q, r, s, t$ are real numbers.

The previous statements imply the results enunciated in the proposition.

Each class $[\mathrm{g}]$ of the left symmetric 2-cohomology of $\mathfrak{e}(2)$ determines a left symmetric product in $\mathfrak{o} \cong \mathbb{R} e_{0} \rtimes_{g} \mathfrak{e}(2)$ by the formula $\left(e_{0}, a\right) \cdot\left(e_{0}, b\right)=(g(a, b), a \cdot b)$, where $g$ is a class representative $[g]$. This method and other observations lead to the following result.

Theorem 2.1. The left symmetric products on the Lie algebra o given by the following tables determine non isomorphic flat left invariant affine connections on the 4-dimensional oscillator group

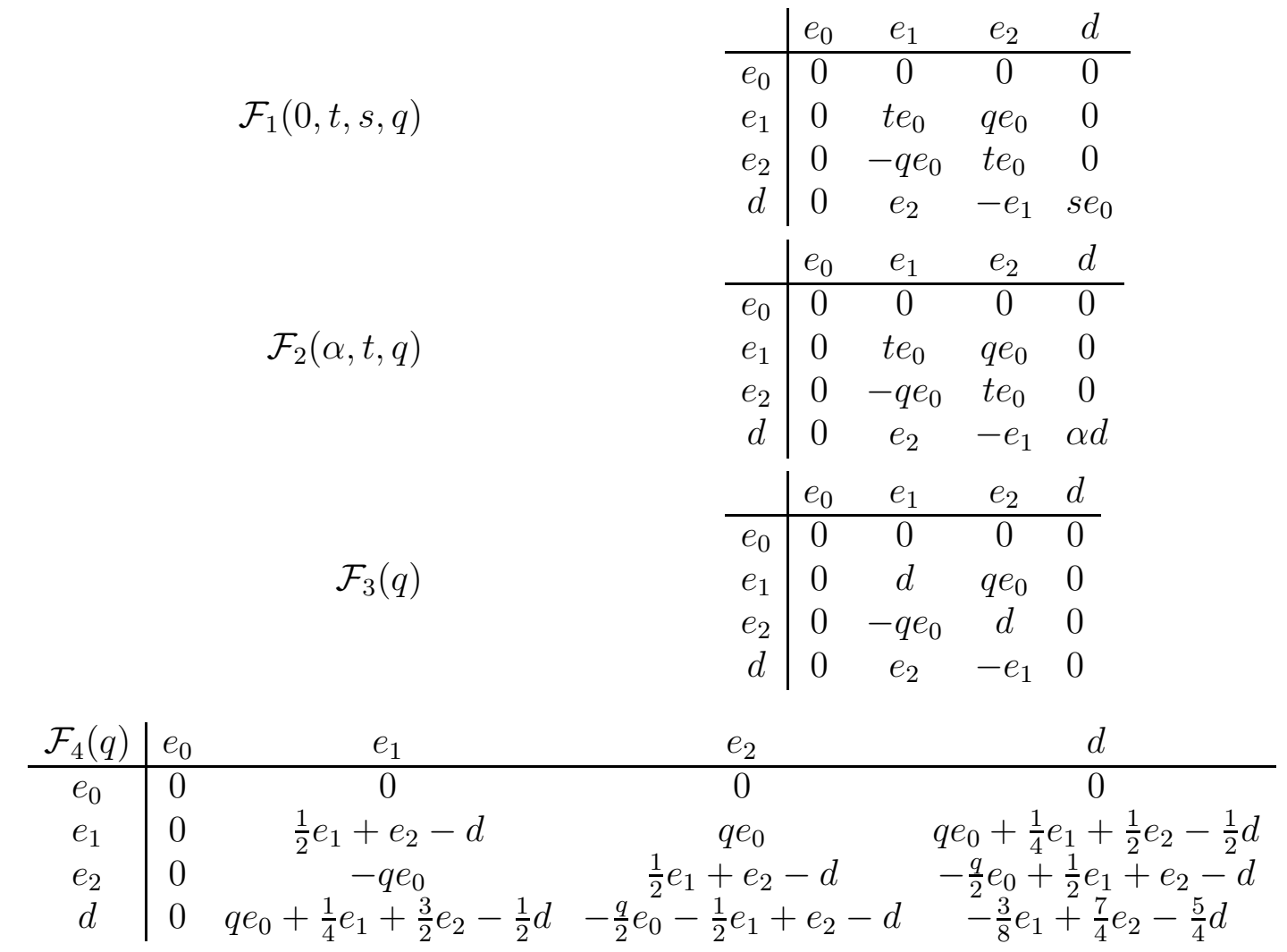

where $t, s \in[0,+\infty), \alpha \in \mathbb{R}^{*}$ and $q=\frac{1}{2}$. 
Corollary 2.1. The flat left invariant affine connections on the 4-dimensional oscillator group given by the products $\mathcal{F}_{1}\left(0, t, s, \frac{1}{2}\right), \mathcal{F}_{3}\left(\frac{1}{2}\right)$ and $\mathcal{F}_{4}\left(\frac{1}{2}\right)$ are geodesically complete.

Proof. This results follows from the fact that each left symmetric algebra has right multiplications $R_{a}$ of trace zero (see [8] and [15]).

Remark 2.1. It is important to notice that left symmetric products $\mathcal{F}_{1}(0, t, s, q), \mathcal{F}_{2}(\alpha, t, q), \mathcal{F}_{3}(q)$ and $\mathcal{F}_{4}(q)$, with $q \neq 0$ are isomorphic respectively to $\mathcal{F}_{1}(0, s, t, 1 / 2), \mathcal{F}_{2}(\alpha, t, 1 / 2), \mathcal{F}_{3}(1 / 2)$ y $\mathcal{F}_{4}(1 / 2)$.

2.3. Double Lie groups of the oscillator group $O$ relative to a solution of classical Yang-Baxter equation. The aim of this section is to determine the dual Lie groups of the oscillator group $O$ relative to solutions of the classical Yang-Baxter equation. We describe the corresponding double Lie groups and we exhibit flat left invariant affine structures on them, by extending the flat affine structures on the oscillator group given in the previous section.

Let $G$ be a real Lie group with Lie algebra $\mathfrak{g}$. A solution of classical Yang-Baxter equation (CYBE) on $G$, is a bivector $r \in \wedge^{2} \mathfrak{g}$ satisfying

$$
[r, r]=0
$$

where $[r, r] \in \wedge^{3} \mathfrak{g}$ is the Schouten bracket (see [6]). The equation (19]) is equivalent to

$$
\alpha\left(\left[r_{\sharp}(\beta), r_{\sharp}(\gamma)\right]\right)+\beta\left(\left[r_{\sharp}(\gamma), r_{\sharp}(\alpha)\right]\right)+\gamma\left(\left[r_{\sharp}(\alpha), r_{\sharp}(\beta)\right]\right)=0,
$$

where $r_{\sharp}: \mathfrak{g}^{*} \rightarrow \mathfrak{g}$ is the linear homomorphism given by $\beta\left(r_{\sharp}(\alpha)\right)=r(\alpha, \beta)$.

Every solution $r$ of CYBE on $G$, determites a Lie algebra structure on the dual vector space $\mathfrak{g}^{*}$ (see [14]), with Lie bracket given by

$$
[\alpha, \beta]_{r}=\operatorname{ad}_{r_{\sharp}(\beta)}^{*} \alpha-\operatorname{ad}_{r_{\sharp}(\alpha)}^{*} \beta
$$

Definition 2.2. The Lie algebra $\mathfrak{g}^{*}(r):=\left(\mathfrak{g}^{*},[,]_{r}\right)$ is called dual Lie algebra of $\mathfrak{g}$ relative to r. We denote by $G^{*}(r)$ a Lie group with Lie algebra $\mathfrak{g}^{*}(r)$.

Notice that, if $\langle$,$\rangle is an orthogonal structure on \mathfrak{g}$, then $\mathfrak{g}^{*}(r)$ has a scalar product given by

$$
\langle\alpha, \beta\rangle^{*}=\left\langle\phi^{-1}(\alpha), \phi^{-1}(\beta)\right\rangle
$$

where $\phi(x)=\langle x$,$\rangle . We denote by k^{*}$ the riemannian pseudo-metric on $G^{*}(r)$ determined by $\langle, \quad\rangle^{*}$ and $\nabla^{*}$ the Levi-Civita connection associated to $k^{*}$.

The essential tool to be used in this section is provided by the following result (see [1]).

Theorem 2.2 (Boucetta-Medina). Let $(G, k)$ be a Lie group endowed with a bi-invariant pseudo-Riemannian metric and let $r$ be a solution of the generalized classical Yang-Baxter equation on $G$. Then, we have the following

(1) $\left(G^{*}(r), k^{*}\right)$ is a locally symmetric pseudo-Riemannian manifold, i.e.,

$$
\nabla^{*} R=0,
$$

where $R$ is the curvature of $k^{*}$. In particular, $R$ vanishes identically when $r$ is a solution of the CYBE.

(2) If $k^{*}$ is flat then it is complete if and only if $G^{*}(r)$ is unimodular.

If the two conditions are verified then $G^{*}(r)$ is solvable. 
Let $r$ be a solution of the CYBE on $G$, then every Lie group of $G^{*}(r)$ is endowed with a flat affine left invariant connection given by

$$
\alpha \cdot \beta:=-\operatorname{ad}_{r_{\sharp}(\alpha)}^{*} \beta
$$

for $\alpha, \beta \in \mathfrak{g}^{*}$ (see for instance [4]). The real vector space $\mathcal{D}(\mathfrak{g}, r):=\mathfrak{g} \oplus \mathfrak{g}^{*}(r)$ endowed with the Lie bracket

$$
[(x, \alpha),(y, \beta)]=\left([x, y]+\operatorname{ad}_{\alpha}^{*} y-\operatorname{ad}_{\beta}^{*} x,[\alpha, \beta]_{r}+\operatorname{ad}_{x}^{*} \beta-\operatorname{ad}_{y}^{*} \alpha\right)
$$

is called the double Lie algebra of Lie algebra $\mathfrak{g}$ relative to $r$. Moreover, it is verified that the scalar product

$$
\langle(x, \alpha),(y, \beta)\rangle:=\beta(x)+\alpha(y)
$$

turns $\mathcal{D}(\mathfrak{g}, r)$ into an orthogonal Lie algebra. It is verified that $\mathfrak{g}$ y $\mathfrak{g}^{*}(r)$ are totally isotropic subalgebras of $\mathcal{D}(\mathfrak{g}, r)$ (see [5]). Therefore, if $\operatorname{dim} \mathfrak{g}=n$, it holds that $\langle$,$\rangle has signature (n, n)$.

Boucetta M. and Medina A. in [1] determined generic solutions of the CYBE of the $\lambda$-oscillator group $O_{\lambda}$ of dimension $n$. In the case $n=4$ such solutions of the CYBE are written as

$$
r=\alpha_{1} e_{0} \wedge e_{1}+\alpha_{2} e_{0} \wedge e_{2}+\alpha_{3} e_{0} \wedge d
$$

for $\alpha_{1}, \alpha_{2}, \alpha_{3} \in \mathbb{R}$. The dual Lie algebra $\mathfrak{o}^{*}(r)=\operatorname{Vect}\left\{e_{0}^{*}, e_{1}^{*}, e_{2}^{*}, d^{*}\right\}$ has non zero Lie brackets (except antisymmetry):

$$
\left[e_{0}^{*}, e_{1}^{*}\right]=-\alpha_{2} d^{*}+\alpha_{3} e_{2}^{*} \quad\left[e_{0}^{*}, e_{2}^{*}\right]=\alpha_{1} d^{*}-\alpha_{3} e_{1}^{*} .
$$

The left symmetric product compatible with the Lie bracket is given by the following products

$$
\begin{gathered}
e_{0}^{*} \cdot e_{0}^{*}=\alpha_{2} e_{1}^{*}-\alpha_{1} e_{2}^{*}, \quad e_{0}^{*} \cdot e_{1}^{*}=-\alpha_{2} d^{*}+\alpha_{3} e_{2}^{*}, \\
e_{0}^{*} \cdot e_{2}^{*}=\alpha_{1} d^{*}-\alpha_{3} e_{1}^{*} .
\end{gathered}
$$

The following proposition describes the dual Lie groups of $O$ relative to solutions of the CYBE.

Lemma 2.2. The simply connected flat affine Lie groups $O^{*}(r)$ corresponding to the Lie algebras $\mathrm{o}^{*}(r)$ where $r=\alpha_{1} e_{0} \wedge e_{1}+\alpha_{2} e_{0} \wedge e_{2}+\alpha_{3} e_{0} \wedge d$ is a solution of the CYBE on $O$, are given by

(1) $O^{*}(r)=\mathbb{R}^{4}$, if $r=0$.

(2) $O^{*}(r)=\mathrm{H}_{3} \times \mathbb{R} \quad$ (direct product), if $r=\alpha_{1} e_{0} \wedge e_{1} \neq 0$ o $r=\alpha_{2} e_{0} \wedge e_{2} \neq 0$.

(3) $O^{*}(r)=\mathbb{R}^{4}$ with product

$$
\begin{aligned}
& (x, y, z, w)\left(x^{\prime}, y^{\prime}, z^{\prime}, w^{\prime}\right)=\left(x+x^{\prime}+(w-z) y^{\prime}+w z^{\prime}+z w^{\prime}, y+y^{\prime}, z+z^{\prime}, w+w^{\prime}\right), \\
& \text { if } \alpha_{3}=0 \text { and } \alpha_{1} \alpha_{2} \neq 0 .
\end{aligned}
$$

(4) $O^{*}(r)=(\mathbb{C} \rtimes \mathbb{R}) \times \mathbb{R}$ with product

$$
(z, s, t)\left(z^{\prime}, s^{\prime}, t^{\prime}\right)=\left(z+e^{i \alpha_{3} s} z^{\prime}, s+s^{\prime}, t+t^{\prime}\right)
$$

if $\alpha_{3} \neq 0$.

Proof. When $\alpha_{1}, \alpha_{2}, \alpha_{3}=0$, we obtain that $\mathfrak{o}^{*}(r)$ is abelian, then $O^{*}(r)=\mathbb{R}^{4}$.

In the second case, the Lie algebra $\mathfrak{o}_{4}^{*}(r)$ is isomorphic to direct sum $\mathfrak{h}_{3} \oplus \mathbb{R} d$, it follows that $O^{*}(r)=\mathrm{H}_{3} \times \mathbb{R}$.

If $\alpha_{3}=0$, the Lie algebra $\mathfrak{o}^{*}(r)$ is isomorphic to Lie algebra

$$
\mathbb{R} d^{*} \times_{g} \mathfrak{m}
$$


central extension of abelian algebra $\mathfrak{m}=\operatorname{Vect}_{\mathbb{R}}\left\{e_{0}^{*}, e_{1}^{*}, e_{2}^{*}\right\}$ by means of the scalar 2-cocicle $g$ of $\mathfrak{m}$ defined by

$$
\begin{aligned}
& g\left(e_{0}^{*}, e_{1}^{*}\right)=-1, \quad g\left(e_{0}^{*}, e_{2}^{*}\right)=1, \\
& g\left(e_{i}^{*}, e_{j}^{*}\right)=0 \quad \text { for }(i, j) \neq(0,1),(0,2) .
\end{aligned}
$$

Then, the Lie group $O^{*}(r)$ with Lie algebra $\mathfrak{o}^{*}(r)$ is $\mathbb{R}^{4}$ with product

$$
(x, y, z, w)\left(x^{\prime}, y^{\prime}, z^{\prime}, w^{\prime}\right)=\left(x+x^{\prime}+(w-z) y^{\prime}+w z^{\prime}+z w^{\prime}, y+y^{\prime}, z+z^{\prime}, w+w^{\prime}\right)
$$

For $\alpha_{3} \neq 0$, we obtain that $\mathfrak{o}^{*}(r)$ is isomorphic to Lie algebra $\operatorname{Vect}_{\mathbb{R}}\left\{f_{0}, f_{1}, f_{2}, d\right\}$

$$
\left[d, f_{1}\right]=\alpha_{3} f_{2}, \quad\left[d, f_{2}\right]=-\alpha_{3} f_{1} .
$$

It follows that

$$
\mathfrak{o}^{*}(r)=\operatorname{Vect}_{\mathbb{R}}\left\{f_{1}, f_{2}\right\} \rtimes_{d} \mathbb{R} d \oplus \mathbb{R} f_{0}
$$

where $d \in \operatorname{End}\left(\operatorname{Vect}_{\mathbb{R}}\left\{f_{1}, f_{2}\right\}\right)$. It verifies that $O^{*}(r)=(\mathbb{C} \rtimes \mathbb{R}) \times \mathbb{R}$ with the product

$$
(s, z, t)\left(s^{\prime}, z^{\prime}, t^{\prime}\right)=\left(s+s^{\prime}, z+e^{i \alpha_{3} s} z^{\prime}, t+t^{\prime}\right) \text {, for all }(s, z, t),\left(s^{\prime}, z^{\prime}, t^{\prime}\right) \in O^{*}(r) .
$$

Theorem 2.3. The Lie groups $O^{*}(r)$ defined by each solution of the CYBE are endowed with a flat left invariant Lorentz metric, a symplectic form and a flat affine left invariant connection.

Proof. Theorem (2.2) implies that $O^{*}(r)$ admits a flat left invariant Lorentz metric. Moreover, this metric is given by the scalar product on $\mathfrak{o}^{*}(r)$ defined by (20) and the left symmetric product is given by (21). It can be verified that the corresponding connection is the Levi-Civita connection.

A symplectic form on Lie group $O^{*}(r)$ is given by the non degenerate scalar 2-cocycle of the algebra $\mathfrak{o}^{*}$ as follows

$$
\omega\left(e_{1}^{*}, e_{2}^{*}\right)=\omega\left(e_{0}^{*}, d^{*}\right)=1 \quad \mathrm{y} \quad \operatorname{Vect}_{\mathbb{R}}\left\{e_{1}^{*}, e_{2}^{*}\right\} \perp_{\omega} \operatorname{Vect}_{\mathbb{R}}\left\{e_{0}^{*}, d^{*}\right\}
$$

for $x, y, z \in \mathfrak{o}^{*}(r)$. The formula

$$
\omega(x y, z)=-\omega(y,[x, z])
$$

defines a left symmetric product compatible with the Lie bracket of $\mathfrak{o}^{*}(r)$.

The non zero products defined by (23) are given by

$$
\begin{array}{ll}
e_{0}^{*} e_{2}^{*}=-\alpha_{3} e_{1}^{*}, & e_{1}^{*} e_{1}^{*}=-\alpha_{3} d^{*}, \\
e_{0}^{*} e_{0}^{*}=-\alpha_{2} e_{2}^{*}-\alpha_{1} e_{0}^{*}, & e_{0}^{*} e_{1}^{*}=\alpha_{3} e_{2}^{*} .
\end{array}
$$

The theorem follows from the above statements.

Remark 2.2. The left symmetric product on $\mathfrak{o}^{*}(r)$ given in (23) defines a complete flat affine left invariant connection on $O^{*}(r)$ which is not compatible with the symplectic form $\omega$.

Remark 2.3 (Double Lie groups of the oscillator). The double Lie algebra of o relative to a solution $r$ of the CYBE given above, correspond to the vector space

$$
\mathcal{D}(\mathfrak{o}, r)=\mathfrak{o}^{*} \oplus \mathfrak{o}=\operatorname{Vect}_{\mathbb{R}}\left\{e_{0}, e_{1} . e_{2}, d, e_{0}^{*}, e_{1}^{*}, e_{2}^{*}, d^{*}\right\}
$$


with Lie brackets (non zero and except antisymmetries)

$$
\begin{aligned}
& {\left[e_{1}, e_{0}^{*}\right]=-\alpha_{3} e_{2}-e_{2}^{*}, \quad\left[e_{2}, e_{0}^{*}\right]=\alpha_{3} e_{1}+e_{1}^{*}, \quad\left[d, e_{0}^{*}\right]=-\alpha_{2} e_{1}+\alpha_{1} e_{2},} \\
& {\left[e_{2}, e_{1}^{*}\right]=-\alpha_{3} e_{0}-d^{*}, \quad\left[d, e_{1}^{*}\right]=\alpha_{2} e_{0}-e_{2}^{*}, \quad\left[e_{1}, e_{2}^{*}\right]=\alpha_{3} e_{0}+d^{*},} \\
& {\left[d, e_{2}^{*}\right]=-\alpha_{1} e_{0}-e_{1}^{*}, \quad\left[e_{0}^{*}, e_{1}^{*}\right]=-\alpha_{2} d^{*}+\alpha_{3} e_{2}^{*}, \quad\left[e_{0}^{*}, e_{2}^{*}\right]=\alpha_{1} d^{*}-\alpha_{3} e_{1}^{*},} \\
& {\left[e_{1}, e_{2}\right]=e_{0}, \quad\left[d, e_{1}\right]=e_{2}, \quad\left[d, e_{2}\right]=-e_{1} \text {. }}
\end{aligned}
$$

\section{Development of Flat AFFine manifold}

This section is devoted to the description of the developing map corresponding to each flat affine left invariant structure on $\operatorname{Aff}(\mathbb{R})$ found in [17] and to each flat affine structure on the oscillator group $O$ exhibited in Section 2. The method we will apply has been introduced by J.L. Koszul in [13].

Proposition 3.1. Let $(M, \nabla)$ be a simply connected flat affine manifold of dimension $n$. Then there exist an affine immersion $D:(M, \nabla) \rightarrow\left(\mathbb{R}^{n}, \nabla^{0}\right)$ and a Lie group homomorphism $A$ : $\operatorname{Aff}(M, \nabla) \rightarrow \operatorname{Aff}\left(\mathbb{R}^{n}, \nabla^{0}\right)$ such that the following diagram commutes

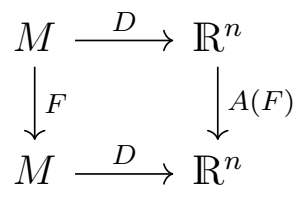

Moreover, $A$ is an immersion.

Proof. Let $p \in M$ and $v \in T_{p}(M)$. For a curve $\alpha$ of class $C^{1}$ on $M$ such that $\alpha(0)=p$ there exist a unique smooth vector field $X(\alpha)$ along $\alpha$ that is parallel relative to $\nabla$ such that $X(\alpha)_{p}=v$. Since $M$ is connected and it has an affine atlas, i.e., the transition maps are local affine transformations of open sets of $\mathbb{R}^{n}$, we can extend $X(\alpha)$ to a smooth vector field $X^{v}$ of $M$ such that $X_{p}^{v}=v$, and $\nabla X^{v}=0$.

Let $\eta$ be a differential 1-form on $M$ with values in $T_{p}(M)$ such that $\eta\left(X^{v}\right)=v$. We shall see that $\eta$ is closed. Notice that

$$
(\nabla \eta)\left(X^{v}, Y\right)=Y\left(\eta\left(X^{v}\right)\right)-\eta\left(\nabla_{Y} X^{v}\right)=0
$$

for all $Y \in \mathfrak{X}(M)$. It follows that $\nabla \eta=0$ and therefore

$$
\begin{aligned}
0 & =(\nabla \eta)(X, Y)-(\nabla \eta)(Y, X) \\
& =Y(\eta(X))-\eta\left(\nabla_{Y} X\right)-X(\eta(Y))+\eta\left(\nabla_{X} Y\right) \\
& =Y(\eta(X))-X(\eta(Y))-\eta\left(\nabla_{Y} X-\nabla_{X} Y\right) \\
& =Y(\eta(X))-X(\eta(Y))-\eta([Y, X]) \\
& =2 \mathrm{~d} \eta(Y, X)
\end{aligned}
$$

for all $X, Y \in \mathfrak{X}(M)$.

Since $M$ is simply connected, there exist a unique differential map $D$ from $M$ in $T_{p}(M)$ such that $\mathrm{d} D=\eta$. In fact, taking a smooth curve $\tau$ in $M$ from $p$ to $q$, it follows from Stokes's theorem that $\int_{\tau} \eta$ does not depend of the curve. Hence the following vector is well defined

$$
D(x)=\int_{p}^{x} \eta .
$$


Now we will show that $D$ is an affine immersion from $M$ in $T_{p}(M)$. Let $\sigma(t)$ be a smooth curve on $M$, since

$$
\dot{\sigma}(t)(\eta(\dot{\sigma}(t)))=\eta\left(\nabla_{\sigma(t)} \dot{\sigma}(t)\right)
$$

it follows that $\sigma$ is a geodesic if and only if $\eta(\dot{\sigma}(t))$ is constant. Then the geodesics of $M$ are curves $\sigma$ such that the map $\eta \circ \sigma$ is constant.

For a geodesic $\sigma(t)$ on $(M, \nabla)$ we have the following

$$
D(\sigma(t))=\int_{p}^{\sigma(0)} \eta+\int_{\sigma(0)}^{\sigma(t)} \eta=\int_{p}^{\sigma(0)} \eta+\int_{\sigma(0)}^{t} \eta(\dot{\sigma}(t)) \mathrm{d} t=\eta(\dot{\sigma}(t)) t+\int_{p}^{\sigma(0)} \eta
$$

Hence, $D(\sigma(t))$ is a line on $T_{p}(M)$, i.e., $D:(M, \nabla) \rightarrow\left(T_{p} M, \nabla^{0}\right)$ is an affine map. Since $\mathrm{d} D=\eta$, the rank of $D$ is constant and equal to $n=\operatorname{dim} M$ in every point, thats means $D$ is an immersion.

Next we will prove that the following map is a Lie group homomorphism

$$
\begin{array}{cl}
A: \operatorname{Aff}(M, \nabla) & \rightarrow \operatorname{Aff}\left(\mathbb{R}^{n}, \nabla^{0}\right) \\
F & \mapsto\left(Q_{F}, L_{F}\right)
\end{array}
$$

First we will see that the map is well defined. Let $L_{F}(v)=u=F_{*, F^{-1}(p)}\left(X_{F^{-1}(p)}^{v}\right)$, with $v \in T_{p}(M)$, and $Q_{F}=D(F(p))$. Since $\nabla F_{*}\left(X^{v}\right)=0$ we have that $F_{*}\left(X^{v}\right)=X^{u}$, which means that $L_{F}$ is $\mathbb{R}$-linear.

Now, we will show that $A(F \circ G)=A(F) \circ A(G)$ for all $F, G \in \operatorname{Aff}(M, \nabla)$.

It directly follows from definition that $L_{F \circ G}=L_{F} \circ L_{G}$. Moreover

$$
\begin{aligned}
Q_{F \circ G} & =D(F(G(p)))=\int_{\tau} \eta, \quad \text { where } \tau \text { is a curve from } p \text { to } F(G(p)) \\
& =\int_{\alpha} \eta+\int_{\alpha^{\prime}} \eta, \quad \text { with } \alpha \text { a curve from } p \text { to } F(p) \\
& \left.=D(F(p))+\int_{F \circ \beta} \eta, \quad \beta \text { is a curve from } \alpha^{\prime} \text { is a curve from } F(p) \text { to } F(G)\right) \\
& =Q_{F}+\int_{\beta} F^{*} \eta
\end{aligned}
$$

On the other hand,

$$
\begin{aligned}
\left(L_{F} \circ \eta\right)_{p}\left(X_{p}^{v}\right) & =L_{F}\left(\eta_{p}\left(X_{p}^{v}\right)\right) \\
& =L_{F}(v) \\
& =u \\
& =\eta_{F(p)}\left(X_{p}^{u}\right) \\
& =\eta_{F(p)}\left(F_{*, p}\left(X_{p}^{v}\right)\right)=\left(F^{*} \eta\right)_{p}\left(X_{p}^{v}\right)
\end{aligned}
$$


It follows that $\int_{\beta} F^{*} \eta=L_{F}\left(Q_{G}\right)$, hence, from (26) we get that $A$ is a group homomorphism. Now notice that for $q \in M$ we have

$$
\begin{aligned}
D(F(q)) & =\int_{\tau} \omega, \quad \text { where } \tau \text { is a curve from } p \text { to } F(q) \\
& =Q_{F}+L_{F}(D(q)) \\
& =A(F)(D(q))
\end{aligned}
$$

that is, (24) is a commutative diagram.

Finally we prove that $A$ is an immersion. First notice that the following diagram is commutative

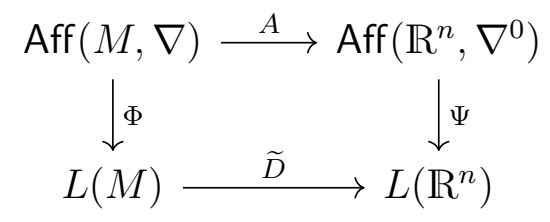

where $\Phi$ maps an affine transformation $F$ of $(M, \nabla)$ in the linear frame $\widetilde{F}\left(u_{0}\right)$ with $u_{0}$ a linear frame of $M$ at $p$ fixed and $\Psi$ maps the affine transformation $T$ of $\left(\mathbb{R}^{n}, \nabla^{0}\right)$ in the linear frame $\widetilde{T}\left(u_{1}\right)$ with $u_{1}=\widetilde{D}\left(u_{0}\right)$ linear frame of $\mathbb{R}^{n}$ at 0 . Since $\Phi$ and $\Psi$ are embeddings (see page 42 [10]), they are, in particular, immersions. Moreover as $D$ is immersion its natural lift is also an immersion. Therefore $A$ is an immersion.

The map $D: M \rightarrow T_{p}(M)$ defined in the previous proposition is called developing map of $(M, \nabla)$ at $p$.

For the following proposition we will consider $M=\operatorname{Aff}(\mathbb{R})_{0}$ with the flat affine structures $\nabla_{2}(\alpha)$ and $\nabla_{1}(\alpha)$ respectively given by $\mathcal{F}_{2}(\alpha)$ and $\mathcal{F}_{1}(\alpha)$ (see [17]).

Proposition 3.2. The corresponding developing maps of $\operatorname{Aff}(\mathbb{R})_{0}$ with values in aff $(\mathbb{R})$ relative to $\nabla_{2}(\alpha)$ and $\nabla_{1}(\alpha)$ are respectively given by

$$
\begin{gathered}
D_{2, \alpha}(x, y)= \begin{cases}\left(\frac{1}{\alpha}\left(x^{\alpha}-1\right), x^{\alpha}(y+1)-\frac{1}{\alpha+1}\left(\alpha x^{\alpha+1}+1\right)\right) & \text { if } \alpha \neq 0,-1 \\
\left(1-\frac{1}{x}, \frac{1+y}{x}-1+\ln x\right) & \text { if } \alpha=-1 \\
(\ln (x), y) & \text { if } \alpha=0\end{cases} \\
D_{1, \alpha}(x, y)= \begin{cases}\left(\frac{1}{\alpha}\left(x^{\alpha}-1\right), y+\frac{1}{\alpha-1}\left(x^{\alpha}-\alpha x\right)+1\right) & \text { if } \alpha \neq 0,1 \\
(x-1, y+1+x(\ln x-1)) & \text { if } \alpha=1\end{cases}
\end{gathered}
$$

Proof. (1) Let $X$ be a smooth vector field on $\operatorname{Aff}(\mathbb{R})_{0}$ such that $\nabla_{2}(\alpha) X=0$. The following identities can be verified

$$
\begin{aligned}
& \nabla_{2}(\alpha)_{e_{1}^{+}} X=0 \\
& \nabla_{2}(\alpha)_{e_{2}^{+}} X=0
\end{aligned}
$$

Solving (29) and (30) with initial conditions $X_{(1,0)}=(a, b) \in \mathfrak{g}$, we have

$$
X=a x^{1-\alpha} \frac{\partial}{\partial x}+(b+\alpha a(x-y-1)) x^{-\alpha} \frac{\partial}{\partial y}
$$


It is easy to verify that the closed 1 -form $\eta$ on $\operatorname{Aff}(\mathbb{R})_{0}$ with values in $\mathfrak{g}$ such that $\eta(X)=(a, b)$, is given by

$$
\eta=\left(x^{\alpha-1} \mathrm{~d} x, \alpha(y-x+1) x^{\alpha-1} \mathrm{~d} x+x^{\alpha} \mathrm{d} y\right)
$$

Taking de curve $\tau(t)=(1+(x-1) t, y t)$, with $t \in[0,1]$, the developing map is written as follows

(a) if $\alpha \neq 0,-1$.

$$
\begin{aligned}
& D_{2, \alpha}(x, y)=\int_{\tau} \eta \\
& =\left(\int_{0}^{1}(1+(x-1) t)^{\alpha-1}(x-1) \mathrm{dt}, \int_{0}^{1} \alpha(x-1)(y-x+1) t(1+(x-1) t)^{\alpha-1}\right. \\
& =\left(\frac{1}{\alpha}\left(x^{\alpha}-1\right), x^{\alpha}(y+1)-\frac{1}{\alpha+1}\left(\alpha x^{\alpha+1}+1\right)\right)
\end{aligned}
$$

(b) For case $\alpha=-1$ :

$$
\begin{aligned}
D_{2,-1}(x, y) & =\int_{\tau} \eta \\
& =\left(\int_{0}^{1} \frac{x-1}{(1+(x-1) t)^{2}} \mathrm{dt}, \int_{0}^{1} \frac{(x-y-1)(x-1) t}{(1+(x-1) t)^{2}} \mathrm{dt}+\frac{y}{1+(x-1) t} \mathrm{dt}\right) \\
& =\left(1-\frac{1}{x}, \frac{1+y}{x}-1+\ln x\right)
\end{aligned}
$$

(c) when $\alpha=0$ :

$$
D_{2,0}(x, y)=(\ln (x), y)
$$

(2) If $X \in \mathfrak{X}\left(\operatorname{Aff}(\mathbb{R})_{0}\right)$ such that $X_{(1,0)}=(a, b) \in \mathfrak{g}$, and

$$
\begin{aligned}
& \nabla_{1}(\alpha) e_{1}^{+} X=0 \\
& \nabla_{1}(\alpha)_{e_{2}^{+}} X=0
\end{aligned}
$$

it can be verified that $X$ is as follows

$$
X= \begin{cases}a x^{1-\alpha} \frac{\partial}{\partial x}+\left(b+\frac{a \alpha}{\alpha-1}\left(x^{1-\alpha}-1\right)\right) \frac{\partial}{\partial y} & \text { si } \alpha \neq 1 \\ a \frac{\partial}{\partial x}+(b-a \ln x) \frac{\partial}{\partial y} & \text { si } \alpha=1\end{cases}
$$

Hence the 1 -form $\eta$ on $\operatorname{Aff}(\mathbb{R})_{0}$ verifying $\eta(X)=(a, b)$, is given by

$$
\eta= \begin{cases}\left(x^{\alpha-1} \mathrm{~d} x, \frac{\alpha}{\alpha-1}\left(x^{\alpha-1}-1\right) \mathrm{d} x+\mathrm{d} y\right) & \text { if } \alpha \neq 1 \\ (\mathrm{~d} x, \ln x \mathrm{~d} x+\mathrm{d} y) & \text { if } \alpha=1\end{cases}
$$


Therefore, the developing map is as follows

$$
\begin{aligned}
D_{1, \alpha}(x, y) & =\int_{\tau} \eta \\
& = \begin{cases}\left(\frac{1}{\alpha}\left(x^{\alpha}-1\right), y+\frac{1}{\alpha-1}\left(x^{\alpha}-\alpha x\right)+1\right) & \text { if } \alpha \neq 0,1 \\
(x-1, y+1+x(\ln x-1)) & \text { if } \alpha=1\end{cases}
\end{aligned}
$$

From now on we will denote by $\nabla_{1}(t, s), \nabla_{2}(\alpha, t), \nabla_{3}$ and $\nabla_{4}$ the flat affine connections on $O$ respectively given by the products $\mathcal{F}_{1}(0, t, s, 1 / 2), \mathcal{F}_{2}(\alpha, t, 1 / 2), \mathcal{F}_{3}(1 / 2)$ y $\mathcal{F}_{4}(1 / 2)$.

Proposition 3.3. The corresponding developing maps of oscillator $O$ relative to the flat affine connections $\nabla_{1}(t, s), \nabla_{2}(\alpha, t), \nabla_{3}$ and $\nabla_{4}$ are respectively

$$
\begin{aligned}
D_{1, t, s}\left(x_{1}, x_{2}, x_{3}, x_{4}\right) & =\left(x_{1}+\frac{t}{2}\left(x_{2}^{2}+x_{3}^{2}\right)+\frac{s}{2} x_{4}^{2}, x_{2}, x_{3}, x_{4}\right) \\
D_{2, \alpha, t}\left(x_{1}, x_{2}, x_{3}, x_{4}\right) & =\left(x_{1}+\frac{t}{2}\left(x_{2}^{2}+x_{3}^{2}\right), x_{2}, x_{3}, \frac{1}{\alpha}\left(e^{\alpha x_{4}}-1\right)\right) \\
D_{3}\left(x_{1}, x_{2}, x_{3}, x_{4}\right) & =\left(x_{1}, x_{2}, x_{3}, x_{4}+\frac{1}{2}\left(x_{2}^{2}+x_{3}^{2}\right)\right) \\
D_{4} & =\left(F_{1}, F_{2}, F_{3}, F_{4}\right)
\end{aligned}
$$

where $F_{i}, i=1,2,3,4$ are detailed at the end of the proof.

Proof. (1) Let $X \in \mathfrak{X}(O)$ such that $X_{(0,0,0,0)}=\left(a_{1}, a_{2}, a_{3}, a_{4}\right)$ with

$$
\begin{aligned}
& \nabla_{2}(\alpha, t)_{e_{i}^{+}} X=0, \quad \text { for } i=0,1,2 \\
& \nabla_{2}(\alpha, t)_{d^{+}} X=0,
\end{aligned}
$$

where $\left(e_{0}^{+}, e_{1}^{+}, e_{2}^{+}, d^{+}\right)$is the basis of left invariant vector fields on $O$ defined in (8). These equations define a system of partial differential equations whose solutions determine the vector field

$$
X=\left(a_{1}-a_{2} t x_{2}-a_{3} t x_{3}\right) \frac{\partial}{\partial x_{1}}+a_{2} \frac{\partial}{\partial x_{2}}+a_{3} \frac{\partial}{\partial x_{3}}+a_{4} e^{-\alpha x_{4}} \frac{\partial}{\partial x_{4}} .
$$

It is easy to verify that the 1 -form $\eta$ such that $\eta(X)=a$ with $a$ in $\mathfrak{o}$, is written

$$
\eta=\left(\mathrm{d} x_{1}+t x_{2} \mathrm{~d} x_{2}+t x_{3} \mathrm{~d} x_{3}, \mathrm{~d} x_{2}, \mathrm{~d} x_{3}, e^{\alpha x_{4}} \mathrm{~d} x_{4}\right)
$$

Hence the developing map is as follows

$$
\begin{aligned}
D_{2, \alpha, t}\left(x_{1}, x_{2}, x_{3}, x_{4}\right) & =\int_{\tau} \eta \\
& =\left(\int_{0}^{1} x_{1} \mathrm{~d} r+t x_{2}^{2} r \mathrm{~d} r+t x_{3}^{2} r \mathrm{~d} r, \int_{0}^{1} x_{2} \mathrm{~d} r, \int_{0}^{1} x_{3} \mathrm{~d} r, \int_{0}^{1} x_{4} e^{\alpha x_{4} r} \mathrm{~d} r\right) \\
& =\left(x_{1}+\frac{t}{2}\left(x_{2}^{2}+x_{3}^{2}\right), x_{2}, x_{3}, \frac{1}{\alpha}\left(e^{\alpha x_{4}}-1\right)\right)
\end{aligned}
$$


(2) The smooth vector field $X$ on $O$ such that $X_{(0,0,0,0)}=\left(a_{1}, a_{2}, a_{3}, a_{4}\right)$ with $\nabla_{1}(t, s) X=0$, can be written as

$$
X=\left(a_{1}-a_{2} t x_{2}-a_{3} t x_{3}-a_{4} s x_{4}\right) \frac{\partial}{\partial x_{1}}+a_{2} \frac{\partial}{\partial x_{2}}+a_{3} \frac{\partial}{\partial x_{3}}+a_{4} \frac{\partial}{\partial x_{4}} .
$$

On the other hand, the 1 -form on $O$ such that $\eta(X)=\left(a_{1}, a_{2}, a_{3}, a_{4}\right) \in \mathfrak{o}$, is given by

$$
\eta=\left(\mathrm{d} x_{1}+t x_{2} \mathrm{~d} x_{2}+t x_{3} \mathrm{~d} x_{3}+s x_{4} \mathrm{~d} x_{4}, \mathrm{~d} x_{2}, \mathrm{~d} x_{3}, \mathrm{~d} x_{4}\right)
$$

Taking a smooth curve $\tau$ on $O$ like $\tau(r)=\left(x_{1} r, x_{2} r, x_{3} r, x_{4} r\right)$ with $r \in[0,1]$, we obtain the developing map

$$
\begin{aligned}
D_{1, t, s}\left(x_{1}, x_{2}, x_{3}, x_{4}\right) & =\int_{\tau} \eta \\
& =\left(\int_{0}^{1} x_{1} \mathrm{~d} r+t x_{2}^{2} r \mathrm{~d} r+t x_{3}^{2} r \mathrm{~d} r+s x_{4}^{2} r \mathrm{~d} r, \int_{0}^{1} x_{2} \mathrm{~d} r, \int_{0}^{1} x_{3} \mathrm{~d} r, \int_{0}^{1} x_{4} \mathrm{~d} r\right) \\
& =\left(x_{1}+\frac{t}{2}\left(x_{2}^{2}+x_{3}^{2}\right)+\frac{s}{2} x_{4}^{2}, x_{2}, x_{3}, x_{4}\right)
\end{aligned}
$$

(3) Let $X$ be a smooth vector field on $O$ satisfying $X_{(0,0,0,0)}=\left(a_{1}, a_{2}, a_{3}, a_{4}\right)$ and

$$
\begin{aligned}
& \nabla_{3, e_{i}^{+}} X=0, \quad \text { para } i=0,1,2 \\
& \nabla_{3, d^{+}} X=0
\end{aligned}
$$

whose solutions determine the vector field

$$
X=a_{1} \frac{\partial}{\partial x_{1}}+a_{2} \frac{\partial}{\partial x_{2}}+a_{3} \frac{\partial}{\partial x_{3}}+\left(a_{4}-a_{2} x_{2}-a_{3} x_{3}\right) \frac{\partial}{\partial x_{4}} .
$$

The 1 -form $\eta$, which $\eta(X)=\left(a_{1}, a_{2}, a_{3}, a_{4}\right) \in \mathfrak{o}$, is written

$$
\eta=\left(\mathrm{d} x_{1}, \mathrm{~d} x_{2}, \mathrm{~d} x_{3}, x_{2} \mathrm{~d} x_{2}+x_{3} \mathrm{~d} x_{3}+\mathrm{d} x_{4}\right)
$$

As a consequence we get that the developing map of $\left(O, \nabla_{3}\right)$ is given by

$$
\begin{aligned}
D_{3}\left(x_{1}, x_{2}, x_{3}, x_{4}\right) & =\int_{\tau} \eta \\
& =\left(\int_{0}^{1} x_{1} \mathrm{~d} r, \int_{0}^{1} x_{2} \mathrm{~d} r, \int_{0}^{1} x_{3} \mathrm{~d} r, \int_{0}^{1} x_{2}^{2} r+x_{3}^{2} r+x_{4} \mathrm{~d} r\right) \\
& =\left(x_{1}, x_{2}, x_{3}, x_{4}+\frac{1}{2}\left(x_{2}^{2}+x_{3}^{2}\right)\right)
\end{aligned}
$$

(4) Finally, we calculate the developing map for $\left(O, \nabla_{4}\right)$. A smooth vector field $X$ on $O$ that is parallel relative to $\nabla_{4}$ with $X_{(0,0,0,0)}=\left(a_{1}, a_{2}, a_{3}, a_{4}\right)$ has the form

$$
\begin{aligned}
X= & \left(-\frac{\hat{a}_{9}}{2} \sin \left(x_{4}\right)+\frac{\hat{a}_{1}}{2} \cos \left(x_{4}\right)-\frac{\hat{a}_{2}}{2} x_{2}-\frac{\hat{a}_{3}}{2} x_{3}+\hat{a}_{4}\right) e_{0}^{+} \\
& +\left(\hat{a}_{5} \sin \left(x_{4}\right)+\hat{a}_{6} \cos \left(x_{4}\right)+\frac{\hat{a}_{3}}{2} x_{2}-\frac{\hat{a}_{2}}{2} x_{3}+\frac{\hat{a}_{4}}{2}\right) e_{1}^{+} \\
& +\left(\hat{a}_{7} \sin \left(x_{4}\right)+\hat{a}_{8} \cos \left(x_{4}\right)+\hat{a}_{3} x_{2}-\hat{a}_{2} x_{3}+\hat{a}_{4}\right) e_{2}^{+} \\
& +\left(\hat{a}_{1} \sin \left(x_{4}\right)+\hat{a}_{9} \cos \left(x_{4}\right)-\hat{a}_{3} x_{2}+\hat{a}_{2} x_{3}-\hat{a}_{4}\right) d^{+}
\end{aligned}
$$


FLAT AFFINE AND SYMPLECTIC GEOMETRIES ON LIE GROUPS

where

$$
\begin{array}{ll}
\hat{a}_{1}=\frac{a_{2}}{2}+a_{3}+\frac{5 a_{4}}{4} & \hat{a}_{2}=a_{3}+a_{4} \\
\hat{a}_{3}=-a_{2}-\frac{a_{4}}{2} & \hat{a}_{4}=a_{1}+\frac{a_{3}}{2}+\frac{a_{4}}{2} \\
\hat{a}_{5}=\frac{1}{2}\left(-\frac{a_{2}}{2}+a_{3}+\frac{3 a_{4}}{4}\right) & \hat{a}_{6}=\frac{1}{2}\left(a_{2}+\frac{a_{3}}{2}+a_{4}\right) \\
\hat{a}_{7}=-\frac{3 a_{2}}{2}-a_{3}-\frac{7 a_{4}}{4} & \hat{a}_{8}=-a_{2}+\frac{3 a_{3}}{2}+a_{4} \\
\hat{a}_{9}=a_{2}-\frac{a_{3}}{2} &
\end{array}
$$

On the other hand, the 1 -form on $O$ with values in $\mathfrak{o}$ such that $\eta(X)=\left(a_{1}, a_{2}, a_{3}, a_{4}\right)$ is $\eta=\left(\omega_{1}, \omega_{2}, \omega_{3}, \omega_{4}\right)$ where

$$
\begin{aligned}
& \omega_{1}=\mathrm{d} x_{1}+\frac{1}{2}\left(\left(1-x_{3}\right) \cos \left(x_{4}\right)+\left(1+x_{2}\right) \sin \left(x_{4}\right)-\frac{1}{2}\right) \mathrm{d} x_{2} \\
& +\frac{1}{2}\left(\left(1+x_{2}\right) \cos \left(x_{4}\right)+\left(x_{3}-1\right) \sin \left(x_{4}\right)-1\right) \mathrm{d} x_{3} \\
& +\frac{1}{2}\left(\left(\frac{3}{2}+x_{2}-\frac{x_{3}}{2}\right) \cos \left(x_{4}\right)+\left(\frac{x_{2}}{2}+x_{3}-\frac{1}{2}\right) \sin \left(x_{4}\right)-\frac{5}{4}\right) \mathrm{d} x_{4}, \\
& \omega_{2}=\frac{1}{2}\left(\left(1+x_{2}\right) \cos \left(x_{4}\right)+\left(\frac{1}{2}+x_{3}\right) \sin \left(x_{4}\right)+1\right) \mathrm{d} x_{2} \\
& +\frac{1}{2}\left(\left(\frac{1}{2}+x_{3}\right) \cos \left(x_{4}\right)-\left(x_{2}+1\right) \sin \left(x_{4}\right)-\frac{1}{2}\right) \mathrm{d} x_{3} \\
& +\frac{1}{2}\left(\left(1+\frac{x_{2}}{2}+x_{3}\right) \cos \left(x_{4}\right)+\left(x_{3}-x_{2}-\frac{3}{4}\right) \sin \left(x_{4}\right)-1\right) \mathrm{d} x_{4}, \\
& \omega_{3}=\left(\left(x_{2}-1\right) \cos \left(x_{4}\right)+\left(\frac{3}{2}+x_{3}\right) \sin \left(x_{4}\right)+1\right) \mathrm{d} x_{2} \\
& +\left(\left(\frac{3}{2}+x_{3}\right) \cos \left(x_{4}\right)+\left(1-x_{2}\right) \sin \left(x_{4}\right)-\frac{1}{2}\right) \mathrm{d} x_{3} \\
& +\left(\left(1+\frac{x_{2}}{2}+x_{3}\right) \cos \left(x_{4}\right)+\left(\frac{x_{3}}{2}-x_{2}+\frac{7}{4}\right) \sin \left(x_{4}\right)-1\right) \mathrm{d} x_{4}, \\
& \omega_{4}=\left(\left(1-x_{2}\right) \cos \left(x_{4}\right)-\left(\frac{1}{2}+x_{3}\right) \sin \left(x_{4}\right)-1\right) \mathrm{d} x_{2} \\
& +\left(\frac{1}{2}-\left(\frac{1}{2}+x_{3}\right) \cos \left(x_{4}\right)+\left(x_{2}-1\right) \sin \left(x_{4}\right)\right) \mathrm{d} x_{3} \\
& +\left(1-\left(\frac{x_{2}}{2}+x_{3}\right) \cos \left(x_{4}\right)+\left(x_{2}-\frac{x_{3}}{2}-\frac{5}{4}\right) \sin \left(x_{4}\right)\right) \mathrm{d} x_{4}
\end{aligned}
$$


Therefore, the corresponding developing map has the following components

$$
\begin{aligned}
& F_{1}\left(x_{1}, x_{2}, x_{3}, x_{4}\right)=x_{1}-\frac{1}{4}\left(x_{2}+5 x_{4}+2\right)-\frac{1}{4 x_{4}}\left(2 x_{3} x_{4}+x_{3}\right) \\
& +\frac{\sin \left(x_{4}\right)}{4 x_{4}^{2}}\left(2 x_{2}^{2}+2 x_{2} x_{4}\left(x_{4}+2\right)+2 x_{3}^{2}-x_{3}\left(x_{4}-6\right) x_{4}+3 x_{4}^{2}\right) \\
& -\frac{\cos \left(x_{4}\right)}{4 x_{4}}\left(2 x_{2}^{2}+2 x_{2} x_{4}+\left(2 x_{3}-1\right)\left(x_{3}+2 x_{4}\right)\right) \\
& F_{2}\left(x_{1}, x_{2}, x_{3}, x_{4}\right)=-\frac{x_{4}}{2}+\frac{\left(x_{2}^{2}+x_{3}^{2}\right)}{2 x_{4}^{2}}\left(\cos \left(x_{4}\right)-1\right)+ \\
& \frac{1}{8}\left(4 x_{2}-2 x_{3}+2\left(x_{2}+2 x_{3}+2\right) \sin \left(x_{4}\right)\right. \\
& \left.+\left(4 x_{2}-4 x_{3}+3\right) \cos \left(x_{4}\right)-3\right) \\
& +\frac{1}{4 x_{4}}\left(\left(2 x_{2}^{2}+x_{3}\left(2 x_{3}+3\right)\right) \sin \left(x_{4}\right)+4 x_{3}\left(\cos \left(x_{4}\right)-1\right)\right) \\
& F_{3}\left(x_{1}, x_{2}, x_{3}, x_{4}\right)=\left(\frac{x_{2}^{2}+x_{3}^{2}}{4 x_{4}^{2}}+\frac{4 x_{2}-2 x_{3}-7}{4}-\frac{x_{2}}{x_{4}}\right) \cos \left(x_{4}\right) \\
& +\left(2 x_{2}^{2}+x_{2}\left(x_{4}-4\right)+2\left(x_{3}\left(x_{3}+x_{4}+2\right)+x_{4}\right)\right) \frac{\sin \left(x_{4}\right)}{2 x_{4}} \\
& -\frac{x_{2}^{2}+x_{3}^{2}}{x_{4}^{2}}+\frac{7-2 x_{3}}{4}+\frac{x_{2}}{x_{4}}+x_{2}-x_{4} \\
& F_{4}\left(x_{1}, x_{2}, x_{3}, x_{4}\right)=\left(\frac{2 x_{3}+5}{4}-\frac{x_{2}^{2}+x_{3}^{2}}{x_{4}^{2}}-x_{2}\right) \cos \left(x_{4}\right) \\
& -\left(\frac{x_{2}^{2}+x_{3}^{2}}{x_{4}}-\frac{2 x_{2}-x_{3}}{x_{4}}+\frac{x_{2}}{2}+x_{3}\right) \sin \left(x_{4}\right) \\
& +\frac{x_{2}^{2}+x_{3}^{2}}{x_{4}^{2}}+\frac{2 x_{3}-5}{4}+x_{4}-x_{2}
\end{aligned}
$$

\section{Symplectic invariant geometry on the Lie group Aff $(\mathbb{R})$}

In what follows we consider the group of affine transformations of real line $G:=\operatorname{Aff}(\mathbb{R})$ as a symplectic Lie group. On the natural coordinates of $G$ the left invariant symplectic form it is written as $\omega^{+}=-\frac{1}{x^{2}} \mathrm{~d} x \wedge \mathrm{d} y$.

Proposition 4.1 ([17]). There exits two left invariant symplectic flat affine, non isomorphic, connections $\nabla_{1}$ and $\nabla_{2}$ on $\operatorname{Aff}(\mathbb{R})$. These connections correspond to the left symmetric products 
on $\mathfrak{g}=\operatorname{LieAff}(\mathbb{R})$ given by the following tables

\begin{tabular}{c|cc} 
& $e_{1}$ & $e_{2}$ \\
\hline$e_{1}$ & $-e_{1}$ & $e_{2}$ \\
$e_{2}$ & 0 & 0 \\
& $e_{1}$ & $e_{2}$ \\
\hline$e_{1}$ & $-\frac{1}{2} e_{1}$ & $\frac{1}{2} e_{2}$ \\
$e_{2}$ & $-\frac{1}{2} e_{2}$ & 0
\end{tabular}

Recall that a symplectomorphism of $(M, \omega)$ is a diffeomorphism $f$ of $M$ such that $f^{*} \omega=\omega$, i.e., a diffeomorphism which preserves the symplectic form $\omega$.

An easy calculation shows the following

Lemma 4.1. A diffeomorphism $f=\left(f_{1}, f_{2}\right)$ of the manifold $\operatorname{Aff}(\mathbb{R})_{0}$ is a symplectomorphism relative to $\omega^{+}$if and only if it verifies the differential equation

$$
\frac{\partial f_{1}}{\partial x} \frac{\partial f_{2}}{\partial y}-\frac{\partial f_{1}}{\partial y} \frac{\partial f_{2}}{\partial x}=\left(\frac{f_{1}}{x}\right)^{2}
$$

In particular the following maps are (functionally independent) symplectomorphism

$$
\begin{aligned}
& f(x, y)=\left(\frac{a x e^{y}}{1+a x e^{y} p(y)}, a e^{y}\right), \text { with } a>0, p(y) \in C^{\infty} \text { and } p>0 . \\
& g(x, y)=\left(e^{y}, \frac{e^{y}}{x}\right) . \\
& h(x, y)=\left(p(x), \frac{\left(h(x)^{2}\right) y}{h^{\prime}(x) x^{2}}+l(x)\right), \text { with } p(x), l(x) \in C^{\infty} \text { and } p>0 .
\end{aligned}
$$

The previous lemma implies the following.

Proposition 4.2. Let $\nabla_{1}$ and $\nabla_{2}$ be the connections given by the products (31) and (32). Then the maps

$$
F(x, y)=\left(a x,-b x^{-1}+a y+d\right)
$$

and

$$
G(x, y)=(a x,-2 b \sqrt{x}+a y+d)
$$

where $a, b, d$ are real numbers with $a>0$, are symplectomorphisms and affine transformations relatively to $\left(G_{0}, \nabla_{1}\right)$ and respectively $\left(G_{0}, \nabla_{2}\right)$.

We finish by computing the Hess connection associated to a Lagrangian bi-foliation of Aff $(\mathbb{R})$ and we show that each of these connections is flat.

Proposition 4.3. There are on $\left(G, \omega^{+}\right)$two transversal Lagrangian foliations given respectively by $\mathcal{F}_{i}=\mathbb{R} e_{i}^{+}, i=1,2$. The Hess connection associated to this bifolation $\left(\mathcal{F}_{1}\right.$ and $\left.\mathcal{F}_{2}\right)$ is a flat affine and Lorentz connection. 
Proof. The formula $\omega(a b, c)=-\omega(b,[a, c])$ defines on the vector space $\mathfrak{g}=\operatorname{aff}(\mathbb{R})$ a left symmetric product compatible with Lie bracket given by following table

\begin{tabular}{c|cc}
$\odot$ & $e_{1}$ & $e_{2}$ \\
\hline$e_{1}$ & $-e_{1}$ & 0 \\
$e_{2}$ & $-e_{2}$ & 0
\end{tabular}

Clearly $\mathbb{R} e_{1}$ and $\mathbb{R} e_{2}$ are transversal Lagrangian Lie subalgebras of $(\mathfrak{g}, \omega)$. Recall that the Hess connection associated to $\mathcal{F}_{1}$ and $\mathcal{F}_{2}$ (see [9] ) is defined by

$$
\nabla_{\left(X_{1}, X_{2}\right)}^{H}\left(Y_{1}, Y_{2}\right)=\left(X_{1} \odot Y_{1}+\left[X_{2}, Y_{1}\right]_{1}, X_{2} \odot Y_{2}+\left[X_{1}, Y_{2}\right]_{2}\right)
$$

where $[,]_{i}$ is the projection on the $i$-foliation and $\odot$ is the product given by (36).

A direct computation shows that this connection correspond to bilinear product on $\mathfrak{g}$ given by

\begin{tabular}{c|cc}
$\star$ & $e_{1}$ & $e_{2}$ \\
\hline$e_{1}$ & $-e_{1}$ & $e_{2}$ \\
$e_{2}$ & 0 & 0
\end{tabular}

From the fact that

$$
L_{e_{1}} \circ L_{e_{2}}-L_{e_{2}} \circ L_{e_{1}}=L_{\left[e_{1}, e_{2}\right]}=L_{e_{2}}
$$

where $L_{e_{i}}$ is the endomorphism $L_{e_{i}}(a)=e_{i} \star a$, it follows that (37) defines a left invariant flat affine connection on $\operatorname{Aff}(\mathbb{R})$.

Moreover, since $\left[e_{1}, e_{2}\right]=e_{1} \star e_{2}-e_{2} \star e_{1}$ we get that the connection $\nabla^{H}$ is torsion free.

Let $\langle$,$\rangle denote the left invariant Lorentz metric on \operatorname{Aff}(\mathbb{R})$ associated to the scalar product on $\mathfrak{g}$ determined by $\left\langle e_{1}, e_{2}\right\rangle=1$ and $\left\langle e_{i}, e_{i}\right\rangle=0, i=1,2$, an easy calculation shows that

$$
\left\langle e_{i} \star e_{j}, e_{k}\right\rangle+\left\langle e_{j}, e_{i} \star e_{k}\right\rangle=0
$$

for all $i, j, k=1,2$. Therefore the Hess connection on $\operatorname{Aff}(\mathbb{R})$ is a Lorentzian connection.

\section{Acknowledgment}

I would like to thank all the members of the Geometry Seminar of the Universidad de Antioquia. They were very helpful at all stages of this work.

\section{REFERENCES}

[1] Boucetta M. And Medina A.; Solutions of the Yang-Baxter equations on quatratic Lie groups: The case of oscillator groups, Journal of geometry and physics 61, 2309-2320, 2011.

[2] Cartan, E., Espaces à connexion affine, projective et conforme, Acta Math. 48 (1926), 1-42.

[3] Dardié J-M And Medina A.; Algèbres de Lie kählériennes et double extension, Journal of Algebra 185, 774-795, 1996.

[4] Diatta A.; Geometrie de Poisson et de Contact des Espaces Homogenes, Directeur A. Medina. These Doctorat. Universite Montpellier, 2000.

[5] Drinfeld V.G.; Hamiltonian structures on Lie groups, Lie bialgebras and the geometric meaning of the clasical Yang-Baxter equations Soviet Math. Dokl. 27, 68-71, 1983.

[6] Dufour, V.G. And Zung, N.T.; Poisson Structures and their Normal Forms, Progress in Mathematics, vol 242, Birkhauser Verlag, Basel, Boston, New York, 2005.

[7] Ehresmann, Les connexions infinitesimales dans un espace fibre differentiable,Colloque de Topologie, Bruxelles (1950), 29-55.

[8] Helmstetter, J.; Radical et groupe formel d'une algèbre symètrique à gauche, Thèse Troisième Cycle, Grenoble, 1975.

[9] Hess, H.; Connexions on Symplectic Manifolds and Geometric Quantization, Differential Geometrical Methods in Math. Physics, Proc. Conf. Aix-en Provence-Salamanca (1979) 135-166.

[10] Kobayashi S.; Tranformation groups in differential geometry Springer-Verlag ISBN: 3-540-58659-8, 1991. 
[11] Kobayashi S, and Nomizu K. Foundations of Differential Geometry, Vol 1. Wiley Classics Library, ISBN: 978-0-471-15733-5, February 1996.

[12] Koszul J., Homologie et cohomologie des algebres de Lie, Bulletin de la Société Mathématique 78 (1950) 65-127.

[13] Koszul J. Variétés localement plates et convexité, Osaka J. Math. 2 (1965) 285-290.

[14] Kosmann-Schwartzbach, Y.; Poisson Drinfe'ld groups, Topics in soliton theory and exactly solvable nonlinear equations, M. Ablowitz, B. Fuchsteiner and M. Kruskal, eds. 191-215, World Scientific Singapore, 1987.

[15] Medina A., Flat left invariant connections adapted to the automorphism structure of a Lie group, J. Differential Geom. 16 (3) (1981) 445-474.

[16] Medina A. And Revoy Ph.; Les grupes oscillateurs et leurs réseaux, Manuscripta Mathematica 52, 81-95, 1985.

[17] Medina A., Saldarriaga O. and Giraldo H. Flat Affine or Projective Geometries on Lie Groups, Journal of Algebra 455 (2016) 183-208.

[18] NiJenhuis A.; Sur une classe de propriétés communes á quelques types différents d'algères, L'enseignement Mathém. XIV, Fasc. 3-4, 225-275.

[19] Shima, H., The Geometry of Hessian Structures, World Scientific Plublishing Co. Pte. Ltd. 2007. 